\title{
The Novel Computer Assisted Film and Television Animation Design Pattern based on Computer Graphics Theory and Three-Dimensional Modeling Structure Optimization
}

\author{
Kangliang $\mathrm{Xiao}^{1, \mathrm{a}}$ \\ ${ }^{1}$ Academy of Fine Arts, GanNan Normal University, 341000, China; \\ axiaokangliang6@sina.com
}

Keywords: Film and Television, Animation Design, Computer Graphics, Structure Optimization.

\begin{abstract}
In this paper, we conduct theoretical analysis on the novel computer assisted film and the television animation design pattern based on the computer graphics theory and three-dimensional modeling structure optimization. Computer graphics rely on itself, the visualization technology of the data and graphics can be effectively combined, graphic display is a major direction in development of computer graphics. To follow the trend, we analyze the issues from different perspectives. Computer graphics applied to computer animation as success of development of flight simulator prompted the application of computer graphics to television, film and advertising industry.
\end{abstract}

\section{Introduction}

Art animation is one of the traditional two-dimensional animations in one of the most infectious form of art as the quality of the art charm of the animation is still widely welcome. But the traditional main method based on key frame animation art, its process is very cumbersome and time-consuming, and require the animators have rich practical experience and the superb painting skills. To change this situation, researchers in recent years, put efforts to develop and explore new method for generating art animation techniques and production. The general approaches could be roughly separated into the list categorizes. (1) Based on traditional 2D animation production process, fusion described the object's 2D half or 3D geometry information to improve automation degree of intermediate frames coloring process. (2) Draw lessons from the current 3D animation production processes and the methods, and combined with the realistic rendering method, improved the traditional two-dimensional animation production method and process of forming new art animation modeling and rendering techniques. (3) Integrated use of computer vision and image processing technology, based on new input information, such as the streaming video to explore new, driven directly by the performance of the art animation production processes and methods to achieve the satisfactory result [1-2].

The development of science and technology to a large extent to promote the fast development of the computer graphics, at the same time, the research and application of graphics to drive the progress and perfection of related disciplines, with the graphics in the role of social and economic development is more and more obvious, it has become an indispensable part of contemporary social progress and development. The relevant technology of the computer graphics could be summarized as the follows according to the literature reviews. (1) Computer science and technology. Computer graphics and the computer are closely related to certain science and technology, computer graphics is produced under the environment of the development of computer technology. (2) Animation technology. Computer animation technology is the most widely used a computer graphics technology, now a lot of animation video and is by the animation modelling technique. Computer animation technology, in a nutshell is to use the advantages of computer itself as the original static image vivid and actualized. (3) Virtual reality technology. Virtual reality technology refers to the screen in the picture presented in the form of a truly which let the audience feel in real life, we watch 3D movies are now using this technology. 
To obtain the advantages of the computer graphics theory to optimize the traditional film and the television animation, in this research, we propose the novel computer assisted film and the television animation design pattern based on the core computer graphics theory and three-dimensional modeling structure optimization. Computer graphics applied to computer animation as success of development of flight simulator prompted the application of computer graphics to television, film and advertising industry. Using the traditional manual animation technology combined with computer graphics can be created with both artistic and technical animation effects, not a single technology can be realized. In the later sub-sections, we will analyze the issues with mathematical modelling and illustration.

\section{Our Proposed Methodology}

\subsection{The Computer based Three-Dimensional Modeling.}

In computer vision, robot visual navigation, automatic testing and assembly and in the field of the medical image analysis, modeling and 3D object recognition plays a vital role. In general, an irregular object can be decomposed into several basic geometries, geometry into the topological relationship between them according to their special objects as the topological relations are important basis to identify the objects in complex environment. Theoretically, the 3D reconstruction problem could be formulized as the equation one for finalizing the modelling procedure [3].

$$
\left(\left(\frac{x}{a_{1}}\right)^{\frac{2}{\varphi_{2}}}+\left(\frac{y}{a_{2}}\right)^{\frac{2}{\varphi_{2}}}\right)^{\frac{\varphi_{2}}{\varphi_{1}}}+\left(\frac{z}{a_{3}}\right)^{\frac{2}{\varphi_{1}}}=1
$$

Practical applications, the model must be unified coordinate system to the environment, to describe whole object environment. Therefore, can be super quadric surface through the coordinate translation and rotation to produce a new form as the model parameters with a total of more than one.

In decomposition and combination method, the decomposition is to split, merge is the true model, as namely the first segmentation modeling again and in the region growing method, segmentation and modeling are synchronized. So the decomposition and combination methods than the region growing method efficiency is low, but it is suitable for irregular data points of 3D modeling and segmentation. The formula two illustrates the transformation procedure of the coordinate system.

$$
\left[\begin{array}{l}
u_{c} \\
v_{c}
\end{array}\right]=\left[\begin{array}{cc}
f_{c x} & 0 \\
0 & f_{c y}
\end{array}\right]\left[\begin{array}{l}
x_{k} \\
y_{k}
\end{array}\right]+\left[\begin{array}{l}
u_{0 c} \\
v_{0 c}
\end{array}\right]
$$

Where the $f$ represents the focal length in the listed formula and this decomposition iteratively, eventually will this group of the data is decomposed into several subsets, the subset of the parts of the object, the decomposition of the integral effect actually. However, if this group of the data points are surrounded by object is an irregular is this rough model fitting parameters is usually local minima, and its solution has great uncertainty which needs further optimization.

\subsection{The Development of Computer Graphics Theory.}

Computer graphics perfect technology mainly through certain concepts and the geometrical way, using computer software to complete the relevant operation steps, as compiled by graphics technology of two-dimensional and three-dimensional technology to realize.

Computer graphics, image difference and connection between them could be analyzed ad achieved from the following perspectives. (1) Graphics and image processing has a certain commonality and dependence between, in practice, if the graphics and the image processing technology combined with using the visual effect of image and quality can be more perfect and clear. (2) Both computer graphics and the image processing, the biggest difference is that the data structure of the computer software is different. Graphics is by these basic elements, such as dot, line, face, therefore, if the simple graphics, so the required data is simple, data quantity is less, relative, graphics and complex, a corresponding increase in the number of data and image processing was conducted for painting of a two-dimensional data set, each pixel is an element of the data set, therefore, it is the amount of data is very large. The 
appearance of the computer-generated animation has its distinctive features, but we can make use of the computer graphics technology to generate photo level image sense of reality [4].

With the progress of the mathematical theory and the pattern discovery approaches, develop path of the computer graphics (CG) theory is enhanced as the following aspects. (1) The chiaroscuro. We will feel in the side of the light intensity change of the brightness of the positive peak effect, on the other side feel the negative peak intensity effect, we will see the stripes on the edge of the image effect and this phenomenon is called Mach band effect. (2) The illumination. Computer generated images belong to the synthetic images, objectively did not exist, as a result of the computer image imaging method with the traditional camera and human visual system imaging methods are similar, therefore, no matter is a physical or mathematical methods, in the process of computer image generation, the observer and observed are indispensable. (3) The material. Computer animation depends on material sense of reality. Material attached on the surface model to add texture and color model.

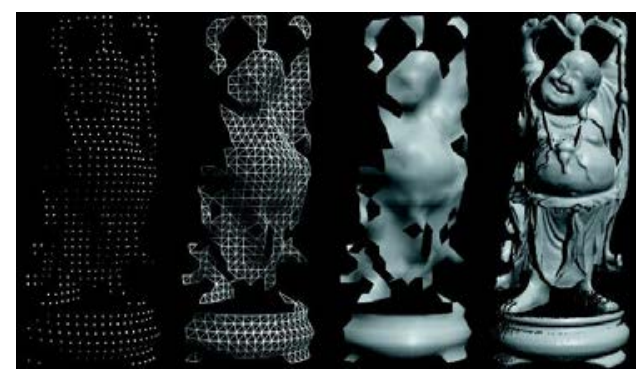

Fig. 2The Illustration of the Computer Graphics Theory Design

\subsection{The Computer Animation Design.}

Computer animation technology is a comprehensive utilization of mathematics, physics, computer graphics, physiology, the product of art and other related discipline knowledge is to use computer to generate a continuous realistic picture. With the development of science and technology, the form of animation can use a computer now generate and play at any time, from making way to ideas, as great changes have taken place in the and its application range is wider.

Based on the target of the design, we could separate the modern animation design patterns into the individual scopes. (1) On the basis of the particle animations. It is mainly to the objective laws of the nature to simulate objects exist. Using the smallest element is the micro particles, through the full life cycle particles to represent series of irregular dynamic characteristics of moving object performance incisively and vividly. (2) Process of animation technology. Usually, animators often like to familiar objects through a simple process of dynamic movement, known as the process of animation. Certain laws or rules established by the motion of mathematical model and function to control the change of the moving object is the most easy to understand the process of animation. (3) The output of computer animation and synthesis. One is the actual image and combination of the three dimensional animation, another is not the need for synthetic technology relatively simple made full use of animation software. (4) Motion capturing technology process. Process capture technology is electronic optics combination of different subjects such as computer graphics, capture technology process for MC technology for short. Usually use the technology to track installation there is tracking device, and then the results obtained by using the computer network. Animators can pick out the useful from the result data to drive the $2 \mathrm{D}$ or $3 \mathrm{D}$ model, making the animation effects [5].

$$
\vec{s}(t)=(1-t) \cdot \vec{p}+t \times \vec{q}
$$

In the study, we found that the length of the monotonicity of the change, especially when the rigid motion morphing not drab had a great influence on animation effects: severe contraction phenomenon leads to the graphics changes is not natural, distortion is serious or severe distortion phenomenon or read in the inner regions of the polygon is distorted, which leads to the middle polygon shape or size to be changed. Therefore, we optimize the tradition ways by using the formula three. 


\subsection{The Novel Computer Assisted Film and Television Animation Design.}

Animation simulation mathematical rules must first clear physical object and its dynamic change process of the simulation animation, again according to the request of animation effects. The whole simulation animation is decomposed into stationary with the movement and display three parts. Show some simulation animation refers to appear gradually along with the change of some parameters of the animation part. The production principle and moving parts are basically the same. Just to draw is generally not erase previous drawing. Achieve physical movement and display of objects. Determine the dynamic coordinates is the key of basic graphics, dynamic coordinate plane rectangular coordinate method is generally used to describe. In each animation scene design, the role of modelling design is the focus of the whole animation, and, in a lot of practice survey found that each cartoon characters all have distinct characteristics, these are all designers cleverly using color way as well as the sketch.

\section{Experiment and Simulation}

To test and verify the performance of the proposed approach, we simulate the concepts with the computer graphic based software. It could be reflected from the result that the general performance of the proposed model is acceptable and describes the detail features of the objects vividly.
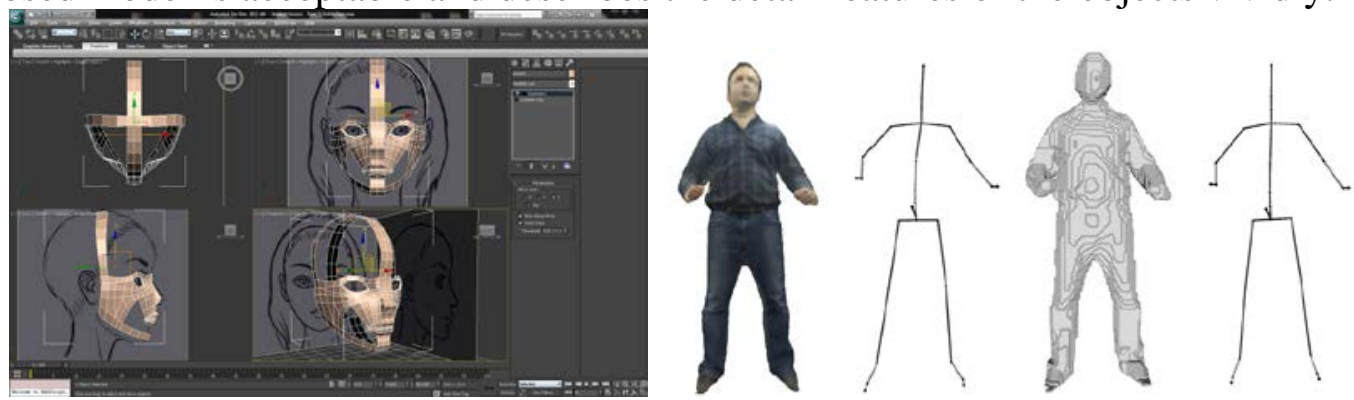

Fig. 2The Simulation Result for the Proposed Computer Assisted Design Pattern

\section{Summary and Conclusion}

In this paper, we conduct theoretical analysis on the novel computer assisted film and the television animation design pattern based on the core computer graphics theory and three-dimensional modeling structure optimization. Basic knowledge of computer graphics and mathematics are closely linked, to some extent, it is the mathematical basis to support the whole the development of computer graphics. Mathematical foundation technology content mainly includes curve surface one of the most critical curve surface theory contains a lot of knowledge, theory of curve surface using its advantages well solved practical some math problems, has been widely recognized and application. We combine the general model to propose the novel design pattern to enhance the industry applications.

\section{References}

[1]. Kodama, Sachiko, et al. "A practical ball sports platform combining dynamic body action with real-time computer graphics during ball play." SIGGRAPH Asia 2015 Emerging Technologies. ACM, 2015.

[2]. Bender, Jan, Kenny Erleben, and Jeff Trinkle. "Interactive simulation of rigid body dynamics in computer graphics." Computer Graphics Forum. Vol. 33. No. 1. 2014.

[3]. Van de Panne, Michiel, and Adam W. Bargteil. "Guest Editor's Introduction: Special Section on the ACM SIGGRAPH/Eurographics Symposium on Computer Animation (SCA)." IEEE transactions on visualization and computer graphics 18.8 (2012): 1189-1190.

[4]. Panozzo, Daniele, et al. "Frame fields: Anisotropic and non-orthogonal cross fields." ACM Transactions on Graphics (TOG) 33.4 (2014): 134.

[5]. Nowozin, Sebastian, and Christoph H. Lampert. "Foundations and Trends ${ }^{\circledR}$ in Computer Graphics and Vision." Foundations and Trends ${ }^{\circledR}$ in Computer Graphics and Vision 6.3-4 (2011). 